\title{
Image Analysis and Machine Learning: How to Foster a Stronger Connection?
}

\author{
Stan Matwin \\ School of Information Technology and Engineering (SITE) \\ University of Ottawa, Canada \\ stan@site.uottawa.ca
}

\begin{abstract}
In this talk I am trying to answer the question stated in its title. I discuss some of the challenges encountered in the use of Machine Learning in image analysis, including examples from our own work. In particular, I look at examples of successful recent research that uses advanced Machine Learning for specific image analysis tasks. I try to generalize lessons learned from this successful research. I argue that some of the reasons for these successes are consistent with the current research trends in Machine Learning. These new approaches resolve some of the difficulties encountered in the past between the two fields, e.g. in the area of feature selection. I look further at some of the recent research trends in Machine Learning (e.g. Active Learning), which might be interesting in image analysis and processing. I also speculate that the new research in cognitive neuroscience might provide interesting cues to Artificial Intelligence in general, and to Computer Vision and image analysis in particular.
\end{abstract}

